\title{
Single-cell RNA-Seq unveils tumor microenvironment
}

\author{
Hae-Ock Lee * E Woong-Yang Park \\ Samsung Genome Institute, Samsung Medical Center, Seoul 06351, Korea
}

\begin{abstract}
Single cell transcriptome analysis is a powerful tool for defining cell types or sub-populations within a heterogeneous bulk population. Tumor-associated microenvironment is a complex ecosystem consisting of numerous cell types that support tumor growth, angiogenesis, immune evasion, and metastasis. With the success of checkpoint inhibitors targeting the immune cell compartment, tumor microenvironment is emerging as a potential anti-cancer target, and understanding it has become an imminent subject in cancer biology. [BMB Reports 2017; 50(6): 283-284]
\end{abstract}

In the article by Chung, Eum, and Lee et al., single-cell RNA-Seq was applied for illustrating the tumor-infiltrating immune cells in primary breast cancer tissues. Briefly, tumor heterogeneity refers to the genetic and non-genetic differences in the tumor cell clone. However, tumor characteristics are highly influenced by the tumor microenvironment, which is reciprocally shaped by the tumor cell itself. Thus, tumor associated microenvironment is a central component of the tumor landscape. Single-cell transcriptome analysis is a robust technique for the characterization of different cell types, enabling the identification of diverse cell types within a tumor microenvironment. In the past, histology best illustrated the diversity of tumor-associated cell types, and the characteristics of each population can be partially elucidated by conventional histology-associated methodology, such as immunohistochemistry. Single cell transcriptomics is potentially the most comprehensive tool for the characterization of tumor landscape, including the tumor cell itself and its associated

${ }^{*}$ Corresponding author. E-mail: haeock.lee@samsung.com

https://doi.org/10.5483/BMBRep.2017.50.6.086

Received 22 May 2017

Keywords: Single-cell RNA-Seq, Tumor microenvironment, Tumorinfiltrating immune cells

Abbreviations: RNA-Seq, RNA sequencing

Perspective to: Woosung Chung, Hye Hyeon Eum, and Hae-Ock Lee et al., 2017, Single-cell RNA-seq enables comprehensive tumor and immune cell profiling in primary breast cancer, Nature Communications (8) 15081 doi: 10.1038 microenvironment. However, there is a critical limit in the application, as cell dissociation required for the current high-throughput analysis of single cell transcriptome is far from perfect. Loss of cell types, adherent cells and rare cells in particular, are the hurdles in comprehensive tumor characterization by single cell transcriptome analysis. Immune cells are non-adherent migrant cells, and are therefore relatively free from loss or damage during cell dissociation. Thus, the current application of single cell transcriptome analysis best captures the tumor associated immune cell compartment in the tumor microenvironment.

Conventionally, characterization of immune cells has relied on surface marker based separation and their bulk transcriptome analysis. This approach is dependent on the prior knowledge of the subpopulation, and is limited by the availability of surface markers and detecting antibodies. In the article by Chung, Eum, and Lee et al., previously defined immune gene sets were employed for the classification of tumor-infiltrating immune cells. Thus, the cell type identification benefitted from prior knowledge of transcriptome data, but were not limited by the few chosen markers. As a result, Chung, Eum, and Lee et al. provided the full landscape of tumor cells and tumor infiltrating immune cells, including $T$ and B lymphocytes and macrophages. Therapeutically, T lymphocytes, macrophages, and tumor cells are all potential targets of immune checkpoint inhibitors. In the study, the checkpoint prevailed on $\mathrm{T}$ lymphocytes, the target that requires to be released from the checkpoint for the activation of anti-tumor immunity. Multiple checkpoint molecules were identified, such as the established PD-1, and more recently developed targets like LAG3 and TIGIT. Considering their expression of cytotoxic mediators, these $\mathrm{T}$ lymphocytes retained their potential to exert anti-tumor immunity. The non-redundant expression of immune checkpoint molecules suggests that a combination of checkpoint inhibitors or screening of the best matching checkpoint inhibitors are necessary for the successful application of immune checkpoint therapy in breast cancer.

This study presented other important findings in intratumoral heterogeneity, such as multiple subtype composition within a patient, which may confer a mechanism for treatment resistance. The most important finding of this study, however, is the demonstration that single cell transcriptome analysis enables comprehensive tumor and immune cell profiling. Expansion of this study to high-throughput single cell trans-

ISSN: 1976-670X (electronic edition)

Copyright (c) 2017 by the The Korean Society for Biochemistry and Molecular Biology

(c) This is an open-access article distributed under the terms of the Creative Commons Attribution Non-Commercial License (http://creativecommons.org/licenses/by-nc/4.0) which permits unrestricted non-commercial use, distribution, and reproduction in any medium, provided the original work is properly cited. 
criptome analysis for a large number of patients, would reveal the full landscape of tumor and associated immune cells in breast cancer with the currently available technology. The full landscape will direct towards better treatment strategies targeting tumor cells and the immune compartment. 